\title{
A culture-sensitive quantitative food frequency questionnaire used in an African population: 1. Development and reproducibility
}

\author{
UE Maclntyre ${ }^{1, *}$, CS Venter ${ }^{2}$ and HH Vorster ${ }^{2}$ \\ 'Department of Paediatrics and Child Health, PO Box 168, Medical University of South Africa, 0204 South \\ Africa: ${ }^{2}$ Department of Nutrition and Family Ecology, Potchefstroom University for Christian Higher Education, \\ Potchefstroom 2520, South Africa
}

Submitted 22 October 1999: Accepted 14 February 2000

\begin{abstract}
Objective: To develop and assess the reproducibility of a quantitative food frequency questionnaire (QFFQ) sensitive to the culture of the African population of the North West Province, South Africa.

Design: A cross-sectional study.

Setting: A community-based field study in a population stratified according to level of urbanization from deep rural to urban upper class.

Subjects: A total of 144 (99 women and 45 men) residents of the North West Province, aged between 15 and 65 years, participated in the study.

Methods: A culture-sensitive, 145-item interviewer-administered QFFQ was designed to cover the whole diet. Portion sizes were estimated from a food portion photograph book (FPPB) showing foods in three portion sizes. The QFFQ was administered twice, 6-12 weeks apart.

Results: Spearman rank correlation coefficients between the two administrations varied from 0.14 for calcium to 0.75 for alcohol. The mean percentage difference between intakes was 8.5 (standard deviation =9.9). Energy, protein, carbohydrate and calcium gave differences within 10\%. Few significant differences among correlation coefficients or percentage difference for gender, age group or strata of urbanization were present. Bland-Altman plots showed significant proportional bias for protein, fibre and vitamin C. More than $70 \%$ of the participants were classified into adjacent quintiles for all nutrients. For food groups, correlation coefficients ranged from 0.25 for milk to 0.45 for vegetable and maize meal groups and $80 \%$ of participants were classified into adjacent quintiles.

Conclusions: The QFFQ appeared to be a reproducible dietary intake assessment instrument.
\end{abstract}

Developing countries are experiencing marked demographic changes, characterized by a shift of the population from rural to urban areas ${ }^{1}$. South Africa, as a semideveloped country and as a result of recent political changes, is experiencing a rapid influx of predominantly African people from the relatively underdeveloped rural areas to the developed, industrialized urban areas ${ }^{2}$. The North West Province typifies this situation, with extremes of affluent and underdeveloped populations and a continuum of stages of transition. The Transition, Health and Urbanization in South Africa (THUSA) study was carried out between 1996 and 1998 to document the effect of urbanization on the health status and dietary intakes of the African population of the North West Province of South Africa. This study aimed at identifying differences in dietary intakes and health status parameters among populations at various levels of urbanization.
Food intakes form part of the complex cultural behaviour of individuals, groups and societies ${ }^{3,4}$. Thus, a dietary assessment tool developed for one population may not be appropriate for use in another. The need for dietary assessment methods sensitive to the culture and food habits of the target population has been highlighted recently by several authors ${ }^{5-7}$. At the time of the THUSA study, no suitable dietary assessment method was available for the study population. Therefore, it was necessary to develop a QFFQ appropriate to the language, culture and food habits of the African population of the North West Province and to test this QFFQ for reproducibility and relative validity.

The purpose of this article is to describe the development and testing of the reproducibility of the culturesensitive QFFQ used in the THUSA study in the North West Province of South Africa. 


\section{Subjects and methods}

\section{The quantitative food frequency questionnaire}

In order to develop a culturally sensitive QFFQ, a sound knowledge of the food habits of the study population must be obtained ${ }^{8}$. Thus, a preliminary study using a combination of in-depth individual interviews and focus group discussions including both quantitative and qualitative methods was undertaken. Participants for the preliminary study were selected from five strata representing the levels of urbanization used in the THUSA study: (i) rural dwellers; (ii) farm workers; (iii) informal settlement dwellers; (iv) middle class urban dwellers; and (v) upper class urban dwellers. In total, 32 adult female volunteers from all strata were recruited by convenience and snowball sampling.

In the first phase of the study, semistructured interviews were conducted by a Tswana (the most common language of the North West Province)-speaking interviewer in the participant's home. Information was collected on the procurement, frequency of consumption, preparation and serving methods of common foods. Actual foods, containers and the participant's own utensils were used to aid the description of food preparation methods and portion sizes. All interviews were tape recorded (with the participant's permission). The tape recordings of the interviews were transcribed and translated into English by an independent translator. This information was coded, the most frequently used foods and recipes identified and food preparation methods compared. For commonly eaten foods, the average amounts of ingredients used in the recipes were calculated. This was done so that the appropriate descriptions were used on the QFFQ and so that local recipes could be added to the food analysis database.

To confirm the initial data collection, the most frequently mentioned foods were prepared by one of the researchers (UEM) following the descriptions of the participants and the average recipes. These dishes were then presented to focus groups of the participants in each area. The same interviewer who had conducted the first interviews acted as the facilitator and the discussions were recorded. Participants discussed the appearance, taste and other characteristics of the dishes, came to an agreement on the ingredients and preparation methods and identified the most commonly consumed foods and drinks in their area. Participants were also asked to demonstrate typical serving sizes of the various foods. These portions were weighed on a scale (EKS, France) and the average weights used to determine the portion sizes for the QFFQ.

Using the results of the preliminary study with existing information on dietary intakes from similar population groups $^{9-12}$, a QFFQ was designed. This was given to five nutritionists, familiar with the food habits of the target population for comment. After improvement, the QFFQ was translated into Tswana by a professional translator and pretested on 10 Tswana speakers for completeness, understandability and appropriateness.

The final QFFQ consisted of 145 food items. Foods were grouped together from the most frequently consumed staple foods (maize) to those foods consumed in small amounts, infrequently (snacks, cakes and sweets). Columns were provided for the amount eaten and the frequency of consumption (number of times per day, per week, per month or seldom).

As an aid for portion size estimation, FPPB was developed using the data obtained in the preliminary study. The FPPB comprised near life-size colour photographs of 37 foods, in three or four portion sizes and photographs of utensils. The development and testing of the FPPB have been described elsewhere ${ }^{13}$.

\section{The reproducibility study}

The first QFFQ interview took place during the data collection of the main THUSA study. Respondents were interviewed by trained fieldworkers in their own language. Respondents estimated portion sizes by indicating the food portion photograph closest in appearance to his or her usual serving size. Besides the FPPB, containers, plates and utensils were used to help participants describe their food intakes.

Volunteers from each stratum of urbanization were recruited for the reproducibility study after the completion of the dietary intake interview. Return visits took place between 6 and 12 weeks after the first interview. Respondents were interviewed using the same procedures as described for the initial interview. A question was added to ascertain if the respondent's circumstances had changed since the first interview. Respondents were randomly assigned to fieldworkers on both the first and repeat interviews. Thus, respondents were not necessarily interviewed by the same fieldworker on both occasions.

The study was approved by the Ethical Committee of the Potchefstroom University for Christian Higher Education. Written informed consent was obtained from all respondents.

\section{Statistical analyses}

Dietary data were analysed using the Food Finder dietary analysis program ${ }^{14}$. Reproducibility was tested for energy, protein, fat, carbohydrate, fibre, calcium, iron and vitamins A and C. All food groups as used in the QFFQ were tested for reproducibility, but only results of the milk, meat, fruit, vegetable, maize meal, bread, fat and sugar groups are reported here.

For both nutrients and food groups, Spearman rank correlation coefficients were calculated, differences between mean intakes were tested by the $t$-test for paired samples and the correlation of the mean of the two administrations of the QFFQ against the difference of the two QFFQs were described by Bland-Altman plots $^{15,16}$. Statistica software ${ }^{17}$ was used for all analyses 
Table 1 Spearman rank correlation coefficients $(R)$ between first and second administrations of the quantitative food frequency questionnaire in the African population of North West Province $(n=144)$

\begin{tabular}{lcc}
\hline Variable & $R$ & $95 \% \mathrm{Cl}$ \\
\hline Energy & 0.28 & 0.12 to 0.42 \\
Protein & 0.31 & 0.15 to 0.45 \\
Carbohydrate & 0.22 & 0.05 to 0.38 \\
Fat & 0.39 & 0.24 to 0.52 \\
Fibre & 0.29 & 0.12 to 0.43 \\
Calcium & 0.14 & -0.23 to 0.29 \\
Iron & 0.28 & 0.12 to 0.42 \\
Vitamin A & 0.34 & 0.19 to 0.48 \\
Vitamin C & 0.38 & 0.23 to 0.51 \\
Alcohol & 0.75 & 0.66 to 0.81 \\
\hline
\end{tabular}

with the significance level set at 5\%. In addition, the distributions of the intakes reported from the two administrations of the QFFQ were divided into quintiles and the number of respondents falling into the same or adjacent quintile on each administration was calculated. The cut-off points for the quintile distributions were calculated for each administration separately.

\section{Results}

The sample for the reproducibility study comprised 144 respondents (99 females and 45 males). Of the total, 24\% (34) of the respondents were from the rural stratum, $14 \%$ (20) from the farm workers' stratum and 20\% (28), $22 \%$ (32) and 21\% (30) from the informal settlement, urban middle class and urban upper class strata, respectively. The proportions of respondents from the strata of urbanization, gender (31\% males and 69\% females) and age groups ( $50 \%$ between 15 and 34.9 years of age) did not differ significantly from those of the main THUSA study $(P>0.05)$. The mean time between the first and second administrations of the QFFQ was 58 ( \pm 27 ) days.

\section{Nutrient intake}

Table 1 shows the Spearman rank correlation coefficients and 95\% confidence intervals (CI) for the nutrients tested. The strongest correlation was for alcohol (0.75) while the weakest and non-significant correlation was for calcium (0.14). The remaining coefficients varied between 0.2 and 0.4 . There were few significant differences among correlation coefficients within the various subgroups (age, gender and urbanization). Although the correlation coefficients obtained for the males tended to be lower than those for the females, the differences were not statistically significant.

Regarding urbanization, the correlation coefficient for alcohol was consistently higher than those obtained for any of the other nutrients tested. With the exceptions of energy, alcohol and fibre, correlation coefficients tended to be lowest for the farm workers' stratum. Significant differences, however, occurred only between the farm workers' stratum and both the rural and the middle class urban strata for calcium $(P<0.01)$. There were no significant differences in correlation coefficients among age groups for any of the nutrients tested $(P>0.05)$.

To test the reproducibility of the QFFQ on a group basis, mean reported intakes for energy and the nutrients tested were compared for the entire sample (Table 2), gender, urbanization and age groups (data not shown). For the entire sample, the mean reported intakes of the second administration of the QFFQ were higher than for the first, with the exceptions of carbohydrate and alcohol.

Table 2 Mean, standard deviation and difference between means of first and second administrations of the quantitative food frequency questionnaire in the African population of North West Province $(n=144)$

\begin{tabular}{lcrrrr}
\hline Nutrient & QFFQ* & Mean & SD & $\begin{array}{c}\text { Difference } \\
(\%) \dagger\end{array}$ & \multicolumn{1}{c}{$95 \% \mathrm{Cl}$} \\
\hline Energy (kJ) & 1 & 8295 & 2882 & $482(5.6)$ & -124 to 1039 \\
Protein (g) & 2 & 8777 & 3304 & & \\
& 1 & 63.0 & 21.4 & $6.6(10.0)$ & 2.0 to 11.2 \\
Carbohydrate (g) & 2 & 69.6 & 26.5 & & \\
& 1 & 297.9 & 122.3 & $-2.3(-0.8)$ & -26.3 to 21.7 \\
Fat (g) & 2 & 295.6 & 114.1 & & \\
& 1 & 56.0 & 25.2 & $10.3(17.0)$ & 4.9 to 15.7 \\
Fibre (g) & 2 & 66.3 & 32.6 & & \\
& 1 & 16.6 & 7.1 & $2.4(13.5)$ & 0.89 to 3.9 \\
Calcium (mg) & 2 & 19.0 & 8.7 & & -43.4 to 75.2 \\
& 1 & 487.1 & 264.8 & $15.9(3.2)$ & \\
Iron (mg) & 2 & 503.0 & 260.2 & & \\
& 1 & 8.5 & 3.9 & $1.2(13.2)$ & -39 to 2.01 \\
Vitamin A (RE) & 2 & 9.7 & 4.1 & & \\
& 1 & 779.9 & 682.6 & $115(13.8)$ & \\
Vitamin C (mg) & 2 & 895.1 & 635.2 & & -0.84 to 27.5 \\
Alcohol (g) & 1 & 54.1 & 78.2 & $13.3(22.0)$ & -3.0 to 1.42 \\
& 2 & 67.4 & 59.2 & & \\
\hline
\end{tabular}

* 1 , first administration of the QFFQ; 2 , second administration of the QFFQ. †Difference $=$ mean of second - mean of first administration of QFFQ; \% mean difference $=($ mean $Q F F Q 2-$ mean QFFQ1)/(QFFQ1 + QFFQ2/2)*100. 
Differences were not significant $(P>0.05)$ for six of the nutrients tested.

The differences expressed as a percentage of the mean of the two administrations of the QFFQ give a clearer indication of the agreement between the results. A difference within $10 \%$ is considered a good agreement ${ }^{18}$. The mean percentage difference for the entire sample was $8.5 \pm$ 9.9\% with energy, protein, carbohydrate and calcium showing differences within 10\%. There appeared to be less agreement among the males, with a mean percentage difference of $13.0 \pm 13.7 \%$ which was significantly higher than the mean percentage difference for the females (8.0 $\pm 6.7 \%)(P<0.05)$.

When the differences in mean intakes between strata were compared by the Kruskal-Wallace test, only the differences for intakes of vitamins $\mathrm{A}$ and $\mathrm{C}$ were statistically significant between the rural and upper class strata and for vitamin $\mathrm{C}$ for the farm and informal settlement strata $(P<0.05)$. Likewise, there were no significant differences among age groups for any of the differences between mean nutrient intakes reported from the first and the second administrations $(P>0.05)$.

Bland-Altman plots (Figs 1 and 2) show the relationship between the difference between the two administrations of the QFFQ and the mean of the two administrations. It is assumed that differences within two standard deviations of the mean difference are in agreement, provided that these differences are not physiologically important ${ }^{15}$. A second aspect of Bland-Altman plots is to show the relationship between the size of the difference and the mean intake of the two administrations of the QFFQ. If proportional bias is present (as the mean intake becomes larger, so does the difference in one direction) the Spearman rank correlation coefficient between the mean intakes and the difference between intakes will be significant ${ }^{15}$. The most desirable state is no proportional bias, narrow limits of agreement and no or very few observations lying outside the limits of agreement ${ }^{15,16}$. As can be seen from Table 3, significant proportional bias was present for protein, fibre and vitamin $\mathrm{C}$, while there was good agreement for energy, protein, carbohydrate, fat, calcium and vitamin C. To illustrate the differences in patterns of agreement, the plots for energy (Fig. 1) and vitamin C (Fig. 2) are shown.

The cumulative percentages of respondents falling into quintiles of the distribution are given in Table 4. The distributions of vitamin $\mathrm{C}$ and alcohol were the most similar on both occasions with $84 \%$ and $75 \%$, respectively, of the sample being classified into the same quintile. The distribution of energy showed the least agreement between administrations with only $22 \%$ of the sample

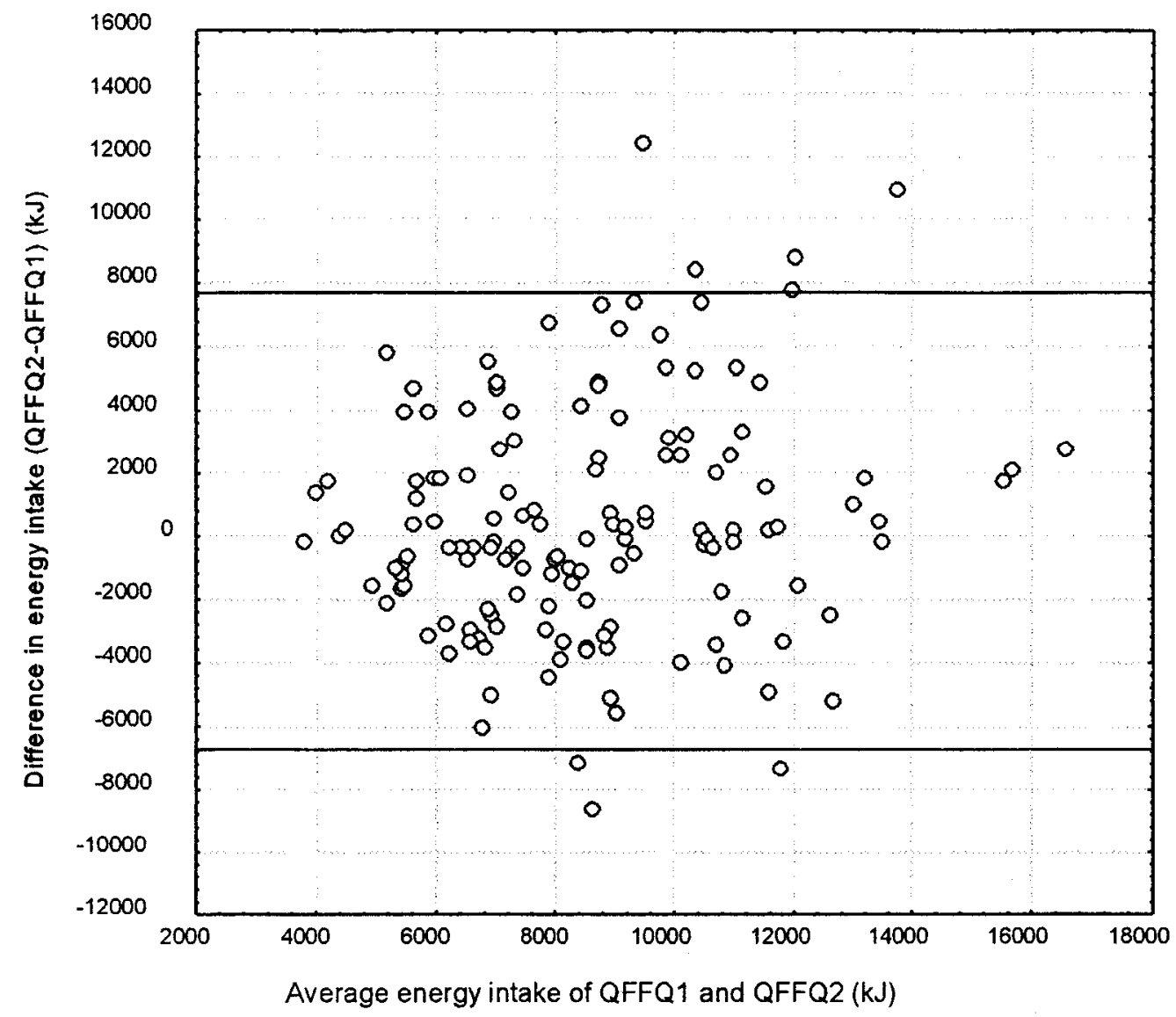

7700

$-6736$

Fig. 1 Bland-Altman plot for energy $(n=144)$. QFFQ1, first administration of the quantitative food frequency questionnaire; QFFQ2, second administration of the quantitative food frequency questionnaire. 


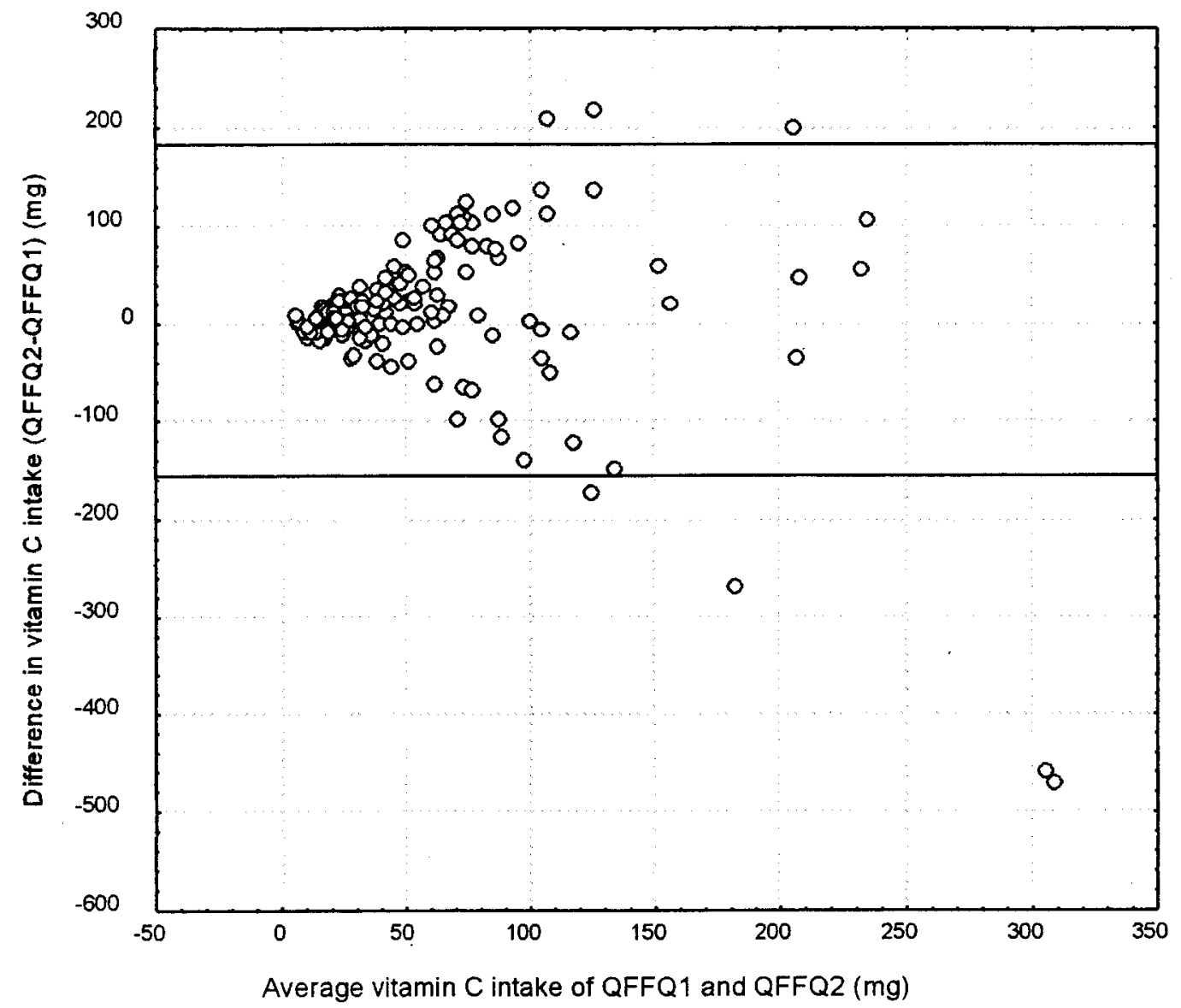

Fig. 2 Bland-Altman plot for vitamin C $(n=144)$. QFFQ1, first administration of the quantitative food frequency questionnaire; QFFQ2, second administration of the quantitative food frequency questionnaire.

being classified into the same quintile and $48 \%$ into adjacent quintiles.

\section{Food intake}

Comparisons of food intakes obtained on the two interviews showed whether respondents reported food intakes consistently. Table 5 shows the results of the

Table 3 Summary of agreement between first and second administrations of the quantitative food frequency questionnaire in the African population of North West Province based on BlandAltman plots $(n=144)$

\begin{tabular}{lcrr}
\hline Nutrient & $\begin{array}{c}\% \\
\text { agreement }\end{array}$ & \multicolumn{1}{c}{$R^{*}$} & \multicolumn{1}{c}{$95 \% \mathrm{Cl}$} \\
\hline Energy (kJ) & 94.5 & 0.14 & -0.03 to 0.30 \\
Protein (g) & 94.5 & 0.23 & 0.07 to 0.38 \\
Carbohydrate (g) & 94.5 & -0.07 & -0.23 to 0.10 \\
Fat $(\mathrm{g})$ & 94.5 & 0.26 & -0.10 to 0.41 \\
Fibre $(\mathrm{g})$ & 94 & 0.15 & 0.02 to 0.30 \\
Calcium (mg) & 94.5 & 0.05 & -0.12 to 0.21 \\
Iron (mg) & 93.1 & 0.10 & -0.07 to 0.26 \\
Vitamin A (RE) & 91.7 & -0.06 & -0.22 to 0.11 \\
Vitamin C (mg) & 95.1 & 0.22 & 0.06 to 0.37 \\
Alcohol (g) & 85.4 & 0.06 & -0.11 to 0.22 \\
\hline
\end{tabular}

* Spearman rank correlation coefficient between the mean of the intakes from the two administrations and the difference of the intakes from the two administrations of the QFFQ.
Spearman rank correlation analyses. All correlation coefficients between the two administrations of the QFFQ were statistically significant. The correlation coefficients for males for two food groups (meat and maize meal) were significantly higher than those for the females $(P<0.05)$.

Mean reported intakes were lower on the first administration of the QFFQ for meat, fruit and vegetables. The reproducibility of the QFFQ appeared to be no

Table 4 Classification of subjects into the same and adjacent quintiles* of distribution for the two administrations of the QFFQ in the African population of North West Province $(n=144)$

\begin{tabular}{lccrr}
\hline & \multicolumn{4}{c}{ Cumulative \% } \\
\cline { 2 - 5 } Variable & $\mathrm{S}$ & \pm 1 & \pm 2 & \pm 3 \\
\hline Energy & 22 & 70 & 93 & 99 \\
Protein & 30 & 82 & 96 & 100 \\
Carbohydrate & 33 & 76 & 96 & 97 \\
Fat & 31 & 77 & 96 & 98 \\
Fibre & 38 & 84 & 99 & 99 \\
Calcium & 31 & 70 & 87 & 93 \\
Iron & 39 & 81 & 96 & 96 \\
Vitamin A & 48 & 85 & 93 & 94 \\
Vitamin C & 84 & 97 & 100 & 100 \\
Alcohol & 75 & 88 & 98 & 99
\end{tabular}

${ }^{*} \mathrm{~S}$, classified into the same quintile; \pm 1 , adjacent quintiles; \pm 2 , two quintiles difference; \pm 3 , three quintiles difference. 
Table 5 Spearman rank correlation coefficients $(R)$ for food groups between the first and second administrations of the quantitative food frequency questionnaire in the African population of North West Province $(n=144)$

\begin{tabular}{lll}
\hline Food group & $R$ & $95 \% \mathrm{Cl}$ \\
\hline Milk & 0.2 & 0.099 to 0.41 \\
Meat & 0.57 & 0.41 to 0.64 \\
Fruit & 0.35 & 0.20 to 0.49 \\
Vegetables & 0.45 & 0.31 to 0.57 \\
Maize meal & 0.45 & 0.31 to 0.57 \\
Bread & 0.29 & 0.13 to 0.43 \\
Fat & 0.32 & 0.17 to 0.46 \\
Sugar & 0.25 & 0.09 to 0.39 \\
\hline
\end{tabular}

different for males and females as no significant differences were found among the mean differences of any of the food groups $(P>0.05)$.

The Bland-Altman plot for maize meal is presented in Fig. 3. There is a significant negative correlation between the difference and mean of the intakes $(r=-0.35 ; 95 \% \mathrm{CI}$ 0.2-0.48). By contrast, the Bland-Altman plot for sugar (Fig. 4) shows no bias in the relationship between reported intake and differences between administrations.

For all food groups, more than $80 \%$ of respondents were classified into the same or adjacent quintiles. There were no significant differences between the proportions of males and females classified into similar quintiles for any of the food groups $(P>0.05)$.

\section{Discussion}

\section{Reproducibility for nutrients}

The range of correlation coefficients (0.14-0.75) (Table 1) obtained in the present study is lower than those reported by other studies with similar intervals (2-4 months) between administrations ${ }^{19-21}$. The range of correlation coefficients obtained in the present study was closest to those obtained for multiethnic youths $(0.26-0.58)^{22}$, Latino mothers $(0.49-0.60)^{23}$ and black males and females $(0.26-0.58)^{24}$. Although lower, the correlation coefficients in the present study show similar trends to those reported by other researchers. The most noticeable similarity is that the highest coefficient was for alcohol (0.75) as has been reported in several other studies ${ }^{18,19,25}$. Munger et al. ${ }^{26}$ have suggested that nutrient intakes derived from only a few foods, such as alcohol, may be less variable and easier for respondents to recall use or abstentions.

As for the differences between the mean reported intakes of the two administrations of the QFFQ (Table 2), only four of the 10 variables tested were significant. Other differences were small, as has been found by other

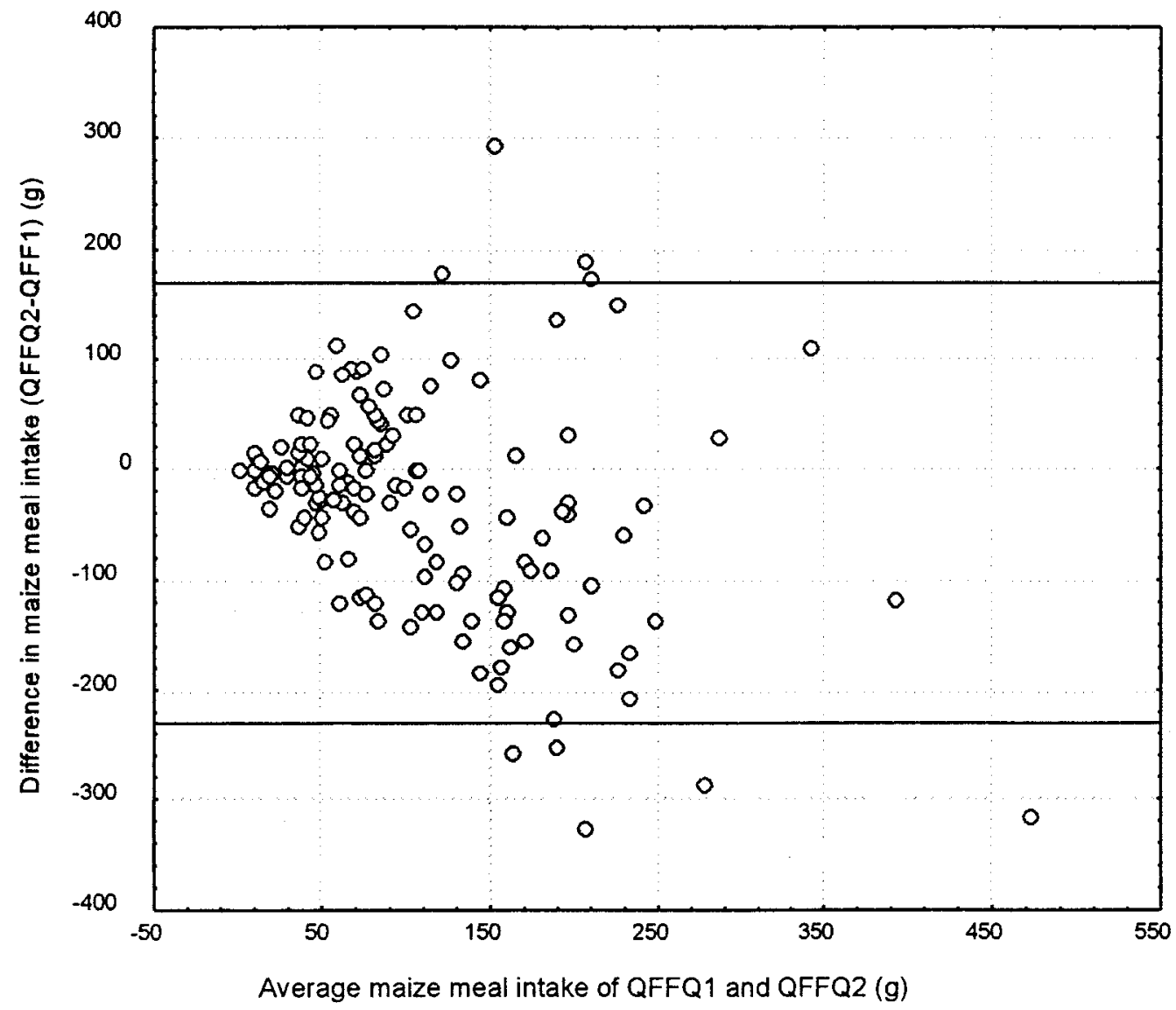

170

Fig. 3 Bland-Altman plot for maize meal $(n=144)$. QFFQ1, first administration of the quantitative food frequency questionnaire; QFFQ2, second administration of the quantitative food frequency questionnaire. 


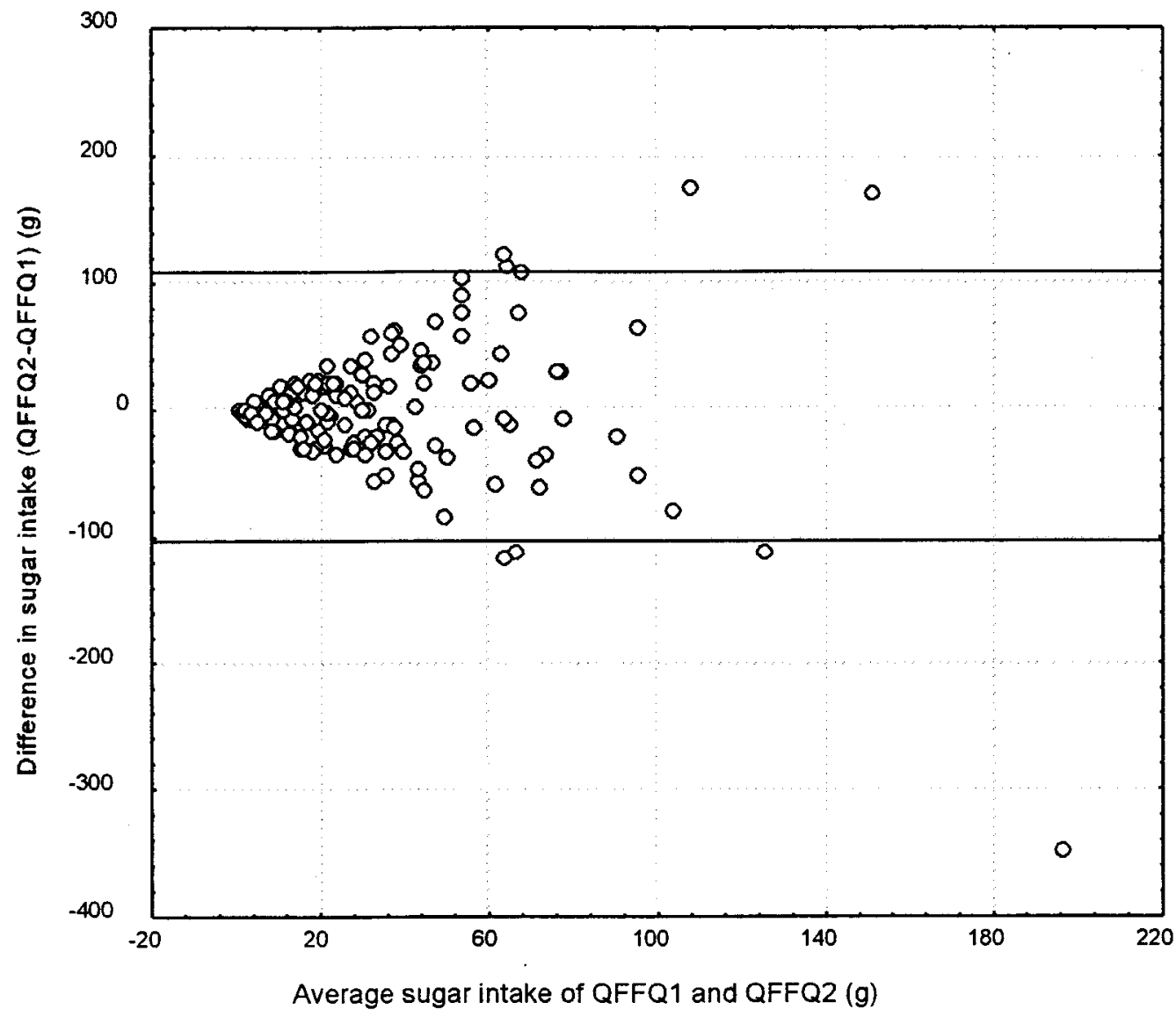

Fig. 4 Bland-Altman plot for sugar $(n=144)$. QFFQ1, first administration of the quantitative food frequency questionnaire; QFFQ2, second administration of the quantitative food frequency questionnaire.

studies $^{18}$. With the exceptions of carbohydrate and alcohol, the mean reported intakes obtained from the second administration were higher than those obtained from the first. This is similar to the trend reported by Männistö et $a l .{ }^{21}$ but differs from several other studies which reported higher mean intakes on the first than on the second measurement ${ }^{18,22,24,26}$.

A factor which is shown by the Bland-Altman method and not by the other analyses is that of proportional bias in the reproducibility of protein, fibre and vitamin C. These nutrients also showed the largest percentage differences between first and second measurements. This suggests that the extent of reproducibility may be influenced by the level of intake, that is, reporting of low intakes may be more reliable than that of high intakes.

The final test of reproducibility was to assess whether the QFFQ was able to place a respondent into the same subgroup of intake on both administrations. Given five quintiles of a distribution, the expected percentages of respondents placed in the same, in the same or within one quintile, or in greater than one quintile by chance alone are $20 \%, 52 \%$ and $48 \%$, respectively ${ }^{27}$. The measurement of energy showed the least ability to classify respondents into the same quintile, with only slightly more respondents than would be expected by chance being placed in the same category (Table 4). For the other nutrients, agreement in the same quintile was higher (mean $=46 \pm 19 \%)$. The percentage of respondents classified within one quintile was considerably higher than would be expected by chance, indicating relatively good agreement. The highest level of agreement was for vitamin C (97\% within one quintile). This finding may explain why vitamin $\mathrm{C}$ showed a relatively good correlation coefficient $(0.38)$ but the highest percentage difference between measurements (22\%). The second interviews were conducted during the citrus season (May-June) when oranges were plentiful in the North West Province. Thus, the entire distribution of vitamin $C$ intake was shifted upwards, resulting in large differences in intakes, but few differences in the ranking of individuals within the distribution.

Comparison of reproducibility measures between subgroups showed few differences. The correlation coefficients for females were slightly higher (but not significantly) than for males (data not shown) while the mean percentage difference for females $(8.0 \pm 6.7 \%)$ was lower than that of the males $(13.0 \pm 3.7 \%)(P<0.05)$. There was no difference in the quintile distribution between genders. Some studies comparing reproducibility of males and 
females have shown no differences ${ }^{28}$ or slightly better reproducibility among males than among females ${ }^{24}$. An important finding in the light of the aims of the THUSA study, that is, the comparison of the effects of urbanization, was that reproducibility was similar across all strata.

\section{Reproducibility of food groups}

According to the Spearman rank correlation coefficients, the meat group showed the highest level of reproducibility $(R=0.57$, Table 5). This correlation coefficient is comparable to the coefficients obtained for meat products in several studies ${ }^{21,27,28}$. The correlation coefficient obtained for vegetables (0.45) was lower than the coefficients reported by Männistö et $a l .^{21}$ and Elmståhl et $a l .^{27}$, but similar to the ranges reported by Ajani et al. ${ }^{29}$ and Riboli et $a l^{30}$. Maize meal showed a reproducibility correlation coefficient (0.45) which was higher than coefficients obtained for rice, samp, bread and other cereals (0.260.33 ) (data not shown). Riboli and co-workers ${ }^{30}$ reported a similar range of reproducibility coefficients for cereals for their long-term study (0.32-0.42) and slightly higher values over the short term $(0.54-0.58)$.

The Bland-Altman plots explain some of the apparently contradictory results obtained using correlation coefficients and differences between means. The plot for maize meal (Fig. 3) shows a significant, negative correlation, suggesting that, at high intake levels, the first administration of the QFFQ produced higher values than the second. Nevertheless, $95 \%$ of the respondents fell within the limits of agreement. Taken together, these results could indicate satisfactory reproducibility of maize meal at low and moderate intakes, but a lessening of agreement at high intake levels.

From the quintile distribution, it seems that the QFFQ was better able to classify respondents into the same category of food group intake on both administrations than it was to classify respondents into the same intake category of nutrients. The percentages of respondents classified into the same quintile of distribution on both administrations (data not shown) were approximately double the percentages obtained for nutrients (Table 4). The cumulative percentage of respondents within one quintile, however, was similar for both nutrients and food groups.

It is difficult to know whether better reproducibility would have been obtained had the same fieldworker conducted the interviews on both occasions. Smith and colleagues $^{31}$ found little difference in reproducibility coefficients when interviews were conducted by the same or different interviewers. Administration by a different fieldworker on the second administration may have advantages. Teufe ${ }^{32}$ has suggested that administration by a different interviewer may reduce the respondent's need to recall earlier responses by removing the feeling of being checked by the same fieldworker. Also, using different fieldworkers gives an indication of the effectiveness of the QFFQ when administered by an unfamiliar interviewer ${ }^{32}$.

It is unlikely that eating patterns would have changed meaningfully during the relatively short interval between administrations. As already pointed out, seasonal changes could have resulted in the higher intakes of oranges and thus of vitamin C. Although the respondents were asked whether any of their circumstances had changed during the intervening period, none answered in the affirmative. Ajani and co-workers ${ }^{29}$, who obtained a similar result, suggested that there may have been changes in eating patterns, but these may have been subtle and not noticed or thought important by the respondents.

In agreement with other studies ${ }^{18,22-24}$, the level of reproducibility varied between nutrients and food groups. Several authors have suggested that frequently consumed foods have better reproducibility than other foods ${ }^{21,29}$. Pietinen et al. ${ }^{33}$ found that reproducibility was generally best for foods eaten infrequently and for foods eaten daily. When all the analyses were taken together, the reproducibility of maize meal intake, the staple food of the population, appeared to be satisfactory. Foods consumed irregularly such as the miscellaneous cereal group, samp and legumes showed relatively low reproducibility. The fact that reproducibility is related to the frequency of consumption of foods is important when comparing results from different populations. A frequently consumed food with good reproducibility in one study may give poor results in another population where it is consumed irregularly.

The important question to be addressed is whether the poor reproducibility of the QFFQ was a result of it not being appropriate to the study population. Teufel ${ }^{32}$ has suggested the following criteria for the design of a culturesensitive dietary intake assessment method: (i) development of specific culturally specific food lists and groups; (ii) creation of a culture-specific database; (iii) definition of culturally appropriate serving sizes; and (iv) comprehensive assessment of the instrument. We believe that the preliminary study and the development of the QFFQ came close to meeting these criteria. Possibly, another method of data collection such as participant observation could have given more representative information. This, however, would have required trained observers, a long study period and financial support, all of which were beyond the resources available for our study. More appropriate food groups may have been achieved by asking the focus groups to group foods from their own perspectives, as suggested by Teufel ${ }^{32}$. A culture-specific database was achieved by using the database developed for use in South Africa ${ }^{14}$ and by adding recipes and local foods to this. Culturally appropriate serving sizes were obtained from both the individual interviews and focus group discussions.

Reproducibility based on the Spearman rank correlation 
coefficients was poor, especially when compared with other published results. Satisfactory reproducibility, however, is also reflected by small differences between the means of each measurement, little proportional bias and a high percentage of respondents classified into adjacant quintiles on both administrations. Thus, alcohol and vitamin A intakes performed well on all measures. For energy and the other nutrients, reproducibility was satisfactory on at least two of the analyses. From the viewpoint of using the QFFQ to compare dietary intakes across urbanization, age and gender, it was reassuring that reproducibility was consistent across all subgroups.

Our results have shown that to assess the reproducibility of a QFFQ, several statistical methods need to be applied to the data. Also, despite very careful preparation and implementation of the QFFQ, reproducibility appeared relatively low. Much research is still needed into obtaining reliable dietary intake data in developing populations. Nutrition researchers in such populations should ask themselves whether dietary assessment methods such as food frequency questionnaires are appropriate or whether new ways of dietary assessment more sensitive to the culture and lifestyles of the populations should be explored.

\section{Acknowledgements}

The study was supported by a grant from the South African Foundation for Research Development. The authors wish to thank the fieldworkers for their invaluable work in collecting the data and the participants in both the preliminary and reproducibility studies for their willing cooperation.

\section{References}

1 Solomons NW, Gross R. Urban nutrition in developing countries. Nutr. Rev. 1995; 53: 90-5.

2 Gelderblom D, Kok P. Urbanisation: South Africa's Challenge. Vol. 1: Dynamics. Pretoria: HSRC Publishers, 1994.

3 Pelto GH. Anthropological contributions to nutrition education research. J. Nutr. Educ. 1981; 13 (Suppl.): S2-8.

4 Ritenbaugh C. An anthropological perspective on nutrition. J. Nutr. Educ. 1981; 13 (Suppl.): S12-15.

5 Buzzard IM, Sievert YA. Research priorities and recommendations for dietary assessment methodology. Am. J. Clin. Nutr. 1994; 59 (Suppl.): S275-80.

6 Coates RJ, Monteilh CP. Assessment of food-frequency questionnaires in minority populations. Am. J. Clin Nutr. 1997; 65 (Suppl.): S1108-15.

7 Romieu I, Hernandez-Avila M, Rivera JA, Ruel MT, Parra S. Dietary studies in countries experiencing a health transition: Mexico and Central America. Am.J. Clin. Nutr. 65 (Suppl.): S1159-65.

8 Hankin JH, Wilkens LR. Development and validation of dietary assessment methods for culturally diverse populations. Am. J. Clin Nutr. 1994; 59 (Suppl.): S198-200.

9 Gresse A. Effects of a traditional African diet on the metabolic control of black patients with type II diabetes mellitus. DSc thesis, Potchefstroom University for Christian Higher Education, Potchefstroom, 1991.
10 Grivetti LE. Kalahari agro-pastoral-hunter-gatherers: the Tswana example. Ecol. Food Nutr. 1979; 84: 235-56.

11 Quin PJ. Food and feeding habits of the Pedi. South Afr. Med.J. 1964; 12: 969-71.

12 Steenkamp A. Food habits of female Tswana agricultural migrants. MSc thesis, University of Stellenbosch, Stellenbosch.

13 Venter CS, MacIntyre UE, Vorster HH. The development and testing of a food portion photograph book for use in an African population. J. Hum. Nutr. Diet. 2000; 13: 205-18.

14 Grant KI, Langenhoven ML, Stockton MA, Day RS, Bauermeister P. Foodfinder dietary analysis software. Release 1.10. 1992. Parowvallei: Medical Research Council, 1992.

15 Bland KM, Altman DG. Comparing two methods of clinical measurement: a personal history. Int. J. Epidemiol. 1995; 24: S7-13.

16 Bland JM, Altman DG. Statistical methods for assessing agreement between two methods of clinical measurement. Lancet 1986; i: $307-11$.

17 Statsoft. Statistica for Windows. Tulsa: Microsoft Corporation, 1995.

18 Wheeler C, Rutishauser L, Conn J, O'Dea K. Reproducibility of a meal-based food frequency questionnaire. The influence of format and time interval between questionnaires. Eur. J. Clin. Nutr. 1994; 48: 795-809.

19 Lindroos A-K, Lissner L, Sjostrom L. Validity and reproducibility of a self administered dietary questionnaire in obese and non-obese subjects. Eur. J. Clin. Nutr. 1993; 47: 461-81.

20 O'Donnell MG, Nelson M, Wise PH, Walker DM. A computerized questionnaire for use in diet health education. 1. Development and validation. Br. J. Nutr. 1991; 66: 315 .

21 Männistö S, Virtanen M, Mikkonen T, Pietinen P. Reproducibility and validity of a food frequency questionnaire in a case-control study on breast cancer. J. Clin. Epidemiol. 1996; 49: 401-9.

22 Rockett HRH, Wolf AM, Colditz GA. Development and reproducibility of a food frequency questionnaire to assess diets of older children and adolescents. J. Am. Diet. Assoc. 1995; 95: 336-40.

23 Basch CE, Shea S, Zybert P. The reproducibility of data from a food frequency questionnaire among low-income Latino mothers and their children. Am. J. Public Health 1994; 84: 861-4.

24 Stevens J, Metcalf PA, Dennis B, Tell GS, Shimakawa T, Folsom AR. Reliability of a food frequency questionnaire by ethnicity, gender, age and education. Nutr. Res. 1996; 16 $735-45$.

25 Goldbohm RA, van t'Veer P, van den Brandt PA, et al Reproducibility of a food frequency questionnaire and stability of dietary habits determined from five annually repeated measurements. Eur. J. Clin. Nutr. 1995; 49: 420-9.

26 Munger RG, Folsom AR, Kushi LH, Kaye SA, Sellers TA. Dietary assessment of older Iowa women with a food frequency questionnaire, nutrient intake, reproducibility, and comparison with 24-hour dietary recall interviews. Am. J. Epidemiol. 1992; 136: 182-200.

27 Elmståhl S, Gullberg B, Riboli E, Saracci R, Lindgårde F. The Malmö food study, the reproducibility of a novel diet history method and an extensive food frequency questionnaire. Eur. J. Clin. Nutr. 1996; 50: 134-42.

28 Kune S, Kune GA, Watson LF. Observations on the reliability and validity of the design and diet history method in the Melbourne colorectal cancer study. Nutr. Cancer 1987; 9: $5-$ 20.

29 Ajani UA, Willett WC, Seddon JM. Reproducibility of a food frequency questionnaire for use in ocular research. Invest. Opthalmol. Vis. Sci. 1994; 35: 2725-33.

30 Riboli E, Toniolo P, Kaaks R, Shore RE, Casagrande C, 
Pasternack BS. Reproducibility of a food frequency questionnaire used in the New York University Women's Health Study: effect of self-selection by study subjects. Eur. J. Clin. Nutr. 1997; 51: 437-42.

31 Smith AF, Jobe B, Mingay DJ. Question-induced cognitive biases in reports of dietary intake by college men and women. Health Psychol. 1991; 10: 244-51.
32 Teufel NI. Development of culturally competent foodfrequency questionnaires. Am. J. Clin. Nutr. 1997; 65 (Suppl.): S1173-8.

33 Pietinen PJ, Hartman AM, Haapa E, et al. Reproducibility and validity of dietary assessment instruments. II. Qualitative food frequency questionnaire. Am.J. Epidemiol. 1988; 128 $667-76$ 